\title{
Some subclasses of multivalent spirallike meromorphic functions
}

Lei Shi ${ }^{* *}$, Zhi-Gang Wang ${ }^{1}$ and Ming-Hua Zeng ${ }^{2}$

Dedicated to Professor Hari M Srivastava

\author{
"Correspondence: \\ shimath@163.com \\ ${ }^{1}$ School of Mathematics and \\ Statistics, Anyang Normal University, \\ Anyang, Henan 455002, People's \\ Republic of China \\ Full list of author information is \\ available at the end of the article
}

\begin{abstract}
In the present paper, we introduce and investigate two new subclasses $\mathcal{M S}_{p}(\alpha, \beta)$ and $\mathcal{M C}_{p}(\alpha, \beta)$ of meromorphic functions. Such results as integral representations and coefficient inequalities are proved. The results presented here would provide extensions of those given in earlier works.

MSC: Primary 30C45; secondary 30C80
\end{abstract}

Keywords: meromorphic functions; meromorphic spirallike functions; differential subordination

\section{Introduction}

Let $\Sigma_{p}$ denote the class of functions $f$ of the form

$$
f(z)=z^{-p}+\sum_{n=1-p}^{\infty} a_{n} z^{n},
$$

which are analytic in the punctured open unit disk

$$
\mathbb{U}^{*}:=\{z: z \in \mathbb{C} \text { and } 0<|z|<1\}=: \mathbb{U} \backslash\{0\} .
$$

Let $\mathcal{P}$ denote the class of functions $p$ given by

$$
p(z)=1+\sum_{n=1}^{\infty} p_{n} z^{n} \quad(z \in \mathbb{U})
$$

which are analytic in $\mathbb{U}$ and satisfy the condition

$$
\Re(p(z))>0 \quad(z \in \mathbb{U})
$$

A function $f \in \Sigma_{p}$ is said to be in the class $\mathcal{M S}_{p}(\alpha)$ of meromorphic $p$-valent starlike functions of order $\alpha$ if it satisfies the inequality

$$
\Re\left(\frac{z f^{\prime}(z)}{f(z)}\right)<-\alpha \quad(z \in \mathbb{U} ; 0 \leqq \alpha<p) .
$$


Moreover, a function $f \in \Sigma_{p}$ is said to be in the class $\mathcal{M K}_{p}(\alpha)$ of meromorphic $p$-valent convex functions of order $\alpha$ if it satisfies the inequality

$$
\Re\left(1+\frac{z f^{\prime \prime}(z)}{f^{\prime}(z)}\right)<-\alpha \quad(z \in \mathbb{U} ; 0 \leqq \alpha<p) .
$$

It is readily verified from (1.2) and (1.3) that

$$
f \in \mathcal{M K}_{p}(\alpha) \Longleftrightarrow-\frac{z f^{\prime}}{p} \in \mathcal{M S}_{p}^{*}(\alpha)
$$

In [1], Wang et al. introduced and investigated two new subclasses of the class $\Sigma_{p}$. A function $f \in \Sigma_{p}$ is said to be in the class $\mathcal{M}_{p}(\beta)$ if it is characterized by the condition

$$
\Re\left(\frac{z f^{\prime}(z)}{f(z)}\right)>-\beta \quad(z \in \mathbb{U} ; \beta>p) .
$$

Also, a function $f \in \Sigma_{p}$ is said to be in the class $\mathcal{N}_{p}(\beta)$ if and only if

$$
\Re\left(1+\frac{z f^{\prime \prime}(z)}{f^{\prime}(z)}\right)>-\beta \quad(z \in \mathbb{U} ; \beta>p) .
$$

Let $\mathcal{A}_{p}$ be the class of functions of the form

$$
f(z)=z^{p}+\sum_{n=p+1}^{\infty} a_{n} z^{n}
$$

which are analytic in $\mathbb{U}$. If it satisfies the condition

$$
\Re\left(e^{i \alpha} \frac{z f^{\prime}(z)}{f(z)}\right)<\beta \quad\left(z \in \mathbb{U} ;-\frac{\pi}{2}<\alpha<\frac{\pi}{2} ; \beta>p \cos \alpha\right),
$$

then we say that $f \in \mathcal{S}_{p}(\alpha, \beta)$. Furthermore, let $\mathcal{C}_{p}(\alpha, \beta)$ denote the subclass of $\mathcal{A}_{p}$ consisting of functions which satisfy the inequality

$$
\Re\left(e^{i \alpha}\left(1+\frac{z f^{\prime \prime}(z)}{f^{\prime}(z)}\right)\right)<\beta \quad\left(z \in \mathbb{U} ;-\frac{\pi}{2}<\alpha<\frac{\pi}{2} ; \beta>p \cos \alpha\right) .
$$

The function classes $\mathcal{S}_{p}(\alpha, \beta)$ and $\mathcal{C}_{p}(\alpha, \beta)$ were introduced and studied recently by Uyanik et al. [2].

Motivated essentially by the above mentioned work, we introduce and investigate the following two subclasses of the class $\Sigma_{p}$ of meromorphic functions.

Definition 1 A function $f \in \Sigma_{p}$ is said to be in the class $\mathcal{M S}_{p}(\alpha, \beta)$ if it satisfies the condition

$$
\Re\left(e^{i \alpha} \frac{z f^{\prime}(z)}{f(z)}\right)>-\beta \quad(z \in \mathbb{U})
$$


for some real $\alpha$ and $\beta$, where (and throughout this paper unless otherwise mentioned) the parameters $\alpha$ and $\beta$ are constrained as follows:

$$
|\alpha|<\frac{\pi}{2} \quad \text { and } \quad \beta>p \cos \alpha
$$

Furthermore, a function $f \in \Sigma_{p}$ is said to be in the class $\mathcal{M C}_{p}(\alpha, \beta)$ if it satisfies the inequality

$$
\Re\left(e^{i \alpha}\left(1+\frac{z f^{\prime \prime}(z)}{f^{\prime}(z)}\right)\right)>-\beta \quad(z \in \mathbb{U}) .
$$

Remark 1 Taking $\alpha=0$, we get the function classes introduced by Wang et al. [1].

Remark 2 We note that $f \in \mathcal{M S}_{p}(\alpha, \beta)$ if and only if

$$
-e^{i \alpha} \frac{z f^{\prime}(z)}{f(z)} \prec \frac{p e^{i \alpha}-\left(2 \beta-p e^{-i \alpha}\right) z}{1-z} .
$$

Also, $f \in \mathcal{M C}_{p}(\alpha, \beta)$ if and only if

$$
-e^{i \alpha}\left(1+\frac{z f^{\prime \prime}(z)}{f^{\prime}(z)}\right) \prec \frac{p e^{i \alpha}-2\left(\beta-p e^{-i \alpha}\right) z}{1-z} .
$$

For some investigations of meromorphic functions, see (for example) the works [1, 3-10] and the references cited in.

In the present paper, we aim at proving some interesting properties such as integral representations and coefficient inequalities of the function classes $\mathcal{M S}_{p}(\alpha, \beta)$ and $\mathcal{M C}_{p}(\alpha, \beta)$.

\section{Main results}

We begin by presenting an integral representation of functions belonging to the class $\mathcal{M S}_{p}(\alpha, \beta)$.

Theorem 1 Let $f \in \mathcal{M S}_{p}(\alpha, \beta)$. Then

$$
f(z)=z^{-p} \cdot \exp \left(2(\beta-p \cos \alpha) e^{-i \alpha} \int_{0}^{z} \frac{\omega(t)}{t(1-\omega(t))} d t\right) \quad\left(z \in \mathbb{U}^{*}\right)
$$

where $\omega$ is analytic in $\mathbb{U}$ with $\omega(0)=0$ and $|\omega(z)|<1$.

Proof For $f \in \mathcal{M S}_{p}(\alpha, \beta)$, we know that (1.6) holds true. It follows that

$$
-e^{i \alpha} \frac{z f^{\prime}(z)}{f(z)}=p e^{i \alpha}-\frac{2(\beta-p \cos \alpha) \omega(z)}{1-\omega(z)}
$$

where $\omega$ is analytic in $\mathbb{U}$ with $\omega(0)=0$ and $|\omega(z)|<1$. We next find from (2.2) that

$$
\frac{f^{\prime}(z)}{f(z)}+\frac{p}{z}=\frac{2(\beta-p \cos \alpha) e^{-i \alpha} \omega(z)}{z(1-\omega(z))} \quad\left(z \in \mathbb{U}^{*}\right)
$$


which, upon integration, yields

$$
\log \left(z^{p} f(z)\right)=2(\beta-p \cos \alpha) e^{-i \alpha} \int_{0}^{z} \frac{\omega(t)}{t(1-\omega(t))} d t .
$$

The assertion (2.1) of Theorem 1 can be easily derived from (2.4).

Note that $f \in \mathcal{M S}_{p}(\alpha, \beta)$ if and only if

$$
-\frac{z f^{\prime}(z)}{p} \in \mathcal{M C}_{p}(\alpha, \beta)
$$

we get the following result.

Corollary 1 Let $f \in \mathcal{M C}_{p}(\alpha, \beta)$. Then

$$
f(z)=-p \int_{z_{0}}^{z} u^{-p-1} \cdot \exp \left(2(\beta-p \cos \alpha) e^{-i \alpha} \int_{0}^{u} \frac{\omega(t)}{t(1-\omega(t))} d t\right) d u \quad\left(z \in \mathbb{U}^{*}\right),
$$

where $\omega$ is analytic in $\mathbb{U}$ with $\omega(0)=0$ and $|\omega(z)|<1$.

Next, we discuss the coefficient estimates of functions belonging to the classes $\mathcal{M S}_{p}(\alpha, \beta)$ and $\mathcal{M C}_{p}(\alpha, \beta)$. The following lemma will be required in the proof of Theorem 2.

Lemma 1 Let $p \in \mathbb{N}$. Suppose also that the sequence $\left\{A_{p+m}\right\}_{m=0}^{\infty}$ is defined by

$$
\begin{cases}A_{p}=\frac{\beta-p \cos \alpha}{p} & (m=0), \\ A_{p+m}=\frac{2(\beta-p \cos \alpha)}{2 p+m}\left(1+\sum_{k=0}^{m-1} A_{p+k}\right) & (m \in \mathbb{N}) .\end{cases}
$$

Then

$$
\begin{aligned}
A_{p+m}= & \frac{2(\beta-p \cos \alpha)}{2 \beta+m+2 p-2 p \cos \alpha} \prod_{k=0}^{m} \frac{2 \beta+k+2 p-2 p \cos \alpha}{2 p+k} \\
& \left(m \in \mathbb{N}_{0}:=\mathbb{N} \cup\{0\}\right) .
\end{aligned}
$$

Proof By virtue of (2.5), we get

$$
(2 p+m+1) A_{p+m+1}=2(\beta-p \cos \alpha)\left(1+\sum_{k=0}^{m} A_{p+k}\right)
$$

and

$$
(2 p+m) A_{p+m}=2(\beta-p \cos \alpha)\left(1+\sum_{k=0}^{m-1} A_{p+k}\right) .
$$

Combining (2.7) and (2.8), we find that

$$
\frac{A_{p+m+1}}{A_{p+m}}=\frac{2 \beta+m+2 p-2 p \cos \alpha}{2 p+m+1} \quad\left(m \in \mathbb{N}_{0}\right) .
$$


Thus,

$$
\begin{aligned}
A_{p+m} & =\frac{A_{p+m}}{A_{p+m-1}} \cdot \frac{A_{p+m-1}}{A_{p+m-2}} \cdots \frac{A_{p+1}}{A_{p}} \cdot A_{p} \\
& =\frac{2 \beta+m-1+2 p-2 p \cos \alpha}{2 p+m} \cdots \frac{2 \beta+2 p-2 p \cos \alpha}{2 p+1} \cdot \frac{2 \beta-2 p \cos \alpha}{2 p} \\
& =\frac{2(\beta-p \cos \alpha)}{2 \beta+m+2 p-2 p \cos \alpha} \prod_{k=0}^{m} \frac{2 \beta+k+2 p-2 p \cos \alpha}{2 p+k} \quad(m \in \mathbb{N}) .
\end{aligned}
$$

The proof of Lemma 1 is thus completed.

Theorem 2 Let $f(z)=z^{-p}+\sum_{m=0}^{\infty} a_{p+m} z^{p+m} \in \mathcal{M S}_{p}(\alpha, \beta)$. Then

$$
\left|a_{p+m}\right| \leqq \frac{2(\beta-p \cos \alpha)}{2 \beta+m+2 p-2 p \cos \alpha} \prod_{k=0}^{m} \frac{2 \beta+k+2 p-2 p \cos \alpha}{2 p+k} \quad\left(m \in \mathbb{N}_{0}\right)
$$

Proof Let

$$
h(z):=\frac{\beta+e^{i \alpha} \frac{z f^{\prime}(z)}{f(z)}+i p \sin \alpha}{\beta-p \cos \alpha} \quad\left(z \in \mathbb{U} ; f \in \mathcal{M S}_{p}(\alpha, \beta)\right) .
$$

We know that $h \in \mathcal{P}$. It follows that

$$
e^{i \alpha} z f^{\prime}(z)=(\beta-p \cos \alpha) f(z) h(z)-(\beta+i p \sin \alpha) f(z) .
$$

Suppose that

$$
h(z)=1+h_{1} z+h_{2} z^{2}+\cdots .
$$

Then

$$
\begin{aligned}
e^{i \alpha}( & \left.-p z^{-p}+p a_{p} z^{p}+(p+1) a_{p+1} z^{p+1}+\cdots+(p+m) a_{p+m} z^{p+m}+\cdots\right) \\
= & (\beta-p \cos \alpha)\left(z^{-p}+a_{p} z^{p}+a_{p+1} z^{p+1}+\cdots\right) \times\left(1+h_{1} z+h_{2} z^{2}+\cdots\right) \\
& -(\beta+i p \sin \alpha)\left(z^{-p}+a_{p} z^{p}+a_{p+1} z^{p+1}+\cdots+a_{p+m} z^{p+m}+\cdots\right) .
\end{aligned}
$$

By evaluating the coefficient of $z^{p+m}$ on both sides of (2.15), we get

$$
\begin{aligned}
e^{i \alpha}(p+m) a_{p+m}= & (\beta-p \cos \alpha)\left(h_{2 p+m}+a_{p} h_{m}+a_{p+1} h_{m-1}+\cdots+a_{p+m}\right) \\
& -(\beta+i p \sin \alpha) a_{p+m} .
\end{aligned}
$$

On the other hand, it is well known that

$$
\left|h_{k}\right| \leqq 2 \quad(k \in \mathbb{N})
$$

From (2.16) and (2.17), we easily get

$$
\left|a_{p}\right| \leqq \frac{\beta-p \cos \alpha}{p}
$$


and

$$
\left|a_{p+m}\right| \leqq \frac{2(\beta-p \cos \alpha)}{2 p+m}\left(1+\sum_{k=0}^{m-1}\left|a_{p+k}\right|\right)
$$

Suppose that $p \in \mathbb{N}$. We define the sequence $\left\{A_{p+m}\right\}_{m=0}^{\infty}$ as follows:

$$
\begin{cases}A_{p}=\frac{\beta-p \cos \alpha}{p} & (m=0) \\ A_{p+m}=\frac{2(\beta-p \cos \alpha)}{2 p+m}\left(1+\sum_{k=0}^{m-1} A_{p+k}\right) & (m \geqq 1) .\end{cases}
$$

In order to prove that

$$
\left|a_{p+m}\right| \leqq A_{p+m} \quad\left(m \in \mathbb{N}_{0}\right)
$$

we use the principle of mathematical induction. It is easy to verify that

$$
\left|a_{p}\right| \leqq A_{p}=\frac{\beta-p \cos \alpha}{p}
$$

Thus, assuming that

$$
\left|a_{p+j}\right| \leqq A_{p+j} \quad\left(j=0,1, \ldots, m ; m \in \mathbb{N}_{0}\right)
$$

we find from (2.19) and (2.23) that

$$
\begin{aligned}
\left|a_{p+m+1}\right| & \leqq \frac{2(\beta-p \cos \alpha)}{2 p+m+1}\left(1+\sum_{k=0}^{m}\left|a_{p+k}\right|\right) \\
& \leqq \frac{2(\beta-p \cos \alpha)}{2 p+m+1}\left(1+\sum_{k=0}^{m}\left|A_{p+k}\right|\right) \\
& =A_{p+m+1} \quad\left(m \in \mathbb{N}_{0}\right) .
\end{aligned}
$$

Therefore, by the principle of mathematical induction, we have

$$
\left|a_{p+m}\right| \leqq A_{p+m} \quad\left(m \in \mathbb{N}_{0}\right)
$$

By means of Lemma 1 and (2.20), we know that

$$
A_{p+m}=\frac{2(\beta-p \cos \alpha)}{2 \beta+m+2 p-2 p \cos \alpha} \prod_{k=0}^{m} \frac{2 \beta+k+2 p-2 p \cos \alpha}{2 p+k} \quad\left(m \in \mathbb{N}_{0}\right) .
$$

Combining (2.25) and (2.26), we readily get the coefficient estimates (2.11) asserted by Theorem 2.

From Theorem 2, we easily get the following result. 
Corollary $2 \operatorname{Let} f(z)=z^{-p}+\sum_{m=0}^{\infty} a_{p+m} z^{p+m} \in \mathcal{M C}_{p}(\alpha, \beta)$. Then

$$
\left|a_{p+m}\right| \leqq \frac{2 p(\beta-p \cos \alpha)}{(p+m)(2 \beta+m+2 p-2 p \cos \alpha)} \prod_{k=0}^{m} \frac{2 \beta+k+2 p-2 p \cos \alpha}{2 p+k} \quad\left(m \in \mathbb{N}_{0}\right)
$$

Remark 3 By setting $\alpha=0$ in Theorem 2, we get the corresponding result due to Wang et al. [1].

Theorem 3 If $f \in \mathcal{M S}_{p}(\alpha, \beta)$, then

$$
\frac{p \cos \alpha-(2 \beta-p \cos \alpha) r}{1-r} \leqq \Re\left(-e^{i \alpha} \frac{z f^{\prime}(z)}{f(z)}\right) \leqq \frac{p \cos \alpha+(2 \beta-p \cos \alpha) r}{1+r}
$$

for $|z|=r<1$.

Proof Consider the function $\varphi$ defined by

$$
\varphi(z):=\frac{p e^{i \alpha}-\left(2 \beta-p e^{-i \alpha}\right) z}{1-z} \quad(z \in \mathbb{U}) .
$$

Let $z=r e^{i \theta}(0<r<1)$, we see that

$$
\Re(\varphi(z))=p \cos \alpha-\frac{2(\beta-p \cos \alpha) r(\cos \theta-r)}{1+r^{2}-2 r \cos \theta} .
$$

Suppose

$$
\psi(t):=p \cos \alpha-\frac{2(\beta-p \cos \alpha) r(t-r)}{1+r^{2}-2 r t} \quad(t:=\cos \theta)
$$

we easily find that

$$
\psi^{\prime}(t)=-2(\beta-p \cos \alpha) \cdot \frac{1-r^{2}}{\left(1+r^{2}-2 r t\right)^{2}}>0
$$

This implies

$$
p \cos \alpha-\frac{2(\beta-p \cos \alpha) r}{1-r} \leqq \Re(\varphi(z)) \leqq p \cos \alpha+\frac{2(\beta-p \cos \alpha) r}{1+r}
$$

which is equivalent to

$$
\frac{p \cos \alpha-(2 \beta-p \cos \alpha) r}{1-r} \leqq \mathfrak{R}(\varphi(z)) \leqq \frac{p \cos \alpha+(2 \beta-p \cos \alpha) r}{1+r}
$$

Noting that $-e^{i \alpha} \frac{z f^{\prime}(z)}{f(z)} \prec \varphi(z)$ and $\varphi(z)$ is univalent in $\mathbb{U}$, we prove the inequality (2.27).

Taking $\alpha=0$ in Theorem 3, we have the following corollary. 
Corollary 3 If $\in \mathcal{M S}_{p}(0, \beta)$, then

$$
\frac{p-(2 \beta-p) r}{1-r} \leqq \Re\left(\frac{z f^{\prime}(z)}{f(z)}\right) \leqq \frac{p+(2 \beta-p) r}{1+r}
$$

for $|z|=r<1$.

Similar to the proof of Theorem 3, we get the following result.

Corollary 4 If $\in \mathcal{M C}_{p}(\alpha, \beta)$, then

$$
\frac{p \cos \alpha-(2 \beta-p \cos \alpha) r}{1-r} \leqq \Re\left(-e^{i \alpha}\left(1+\frac{z f^{\prime \prime}(z)}{f^{\prime}(z)}\right)\right) \leqq \frac{p \cos \alpha+(2 \beta-p \cos \alpha) r}{1+r}
$$

for $|z|=r<1$.

Corollary 5 If $\in \mathcal{M C}_{p}(0, \beta)$, then

$$
\frac{p-(2 \beta-p) r}{1-r} \leqq \Re\left(1+\frac{z f^{\prime \prime}(z)}{f^{\prime}(z)}\right) \leqq \frac{p+(2 \beta-p) r}{1+r}
$$

for $|z|=r<1$.

Now, we present some sufficient conditions for functions belonging to the classes $\mathcal{M S}_{p}(\alpha, \beta)$ and $\mathcal{M C}_{p}(\alpha, \beta)$.

Theorem 4 If $\in \mathcal{M S}_{p}(\alpha, \beta)$ satisfies the condition

$$
\sum_{n=1-p}^{\infty}\left(\left|n e^{i \alpha}+\lambda\right|+\left|n e^{i \alpha}+2 \beta-\lambda\right|\right)\left|a_{n}\right| \leqq\left|p e^{i \alpha}-2 \beta+\lambda\right|-\left|p e^{i \alpha}-\lambda\right|
$$

for some real $\alpha, \beta$ and $\lambda(0 \leqq \lambda \leqq p \cos \alpha)$, then $f \in \mathcal{M S}_{p}(\alpha, \beta)$.

Proof To prove $f \in \mathcal{M S}_{p}(\alpha, \beta)$, it suffices to show that

$$
\left|\frac{e^{i \alpha} \frac{z f^{\prime}(z)}{f(z)}+\lambda}{e^{i \alpha} \frac{z f^{\prime}(z)}{f(z)}+(2 \beta-\lambda)}\right|<1 \quad(z \in \mathbb{U} ; 0 \leqq \lambda \leqq p \cos \alpha) .
$$

From (2.34), we know that

$$
\left|p e^{i \alpha}-2 \beta+\lambda\right|-\sum_{n=1-p}^{\infty}\left|n e^{i \alpha}+2 \beta-\lambda\right|\left|a_{n}\right| \geqq\left|p e^{i \alpha}-\lambda\right|+\sum_{n=1-p}^{\infty}\left|n e^{i \alpha}+\lambda\right|\left|a_{n}\right|>0
$$


Now, by the maximum modulus principle, we deduce from (1.1) and (2.36) that

$$
\begin{aligned}
\left|\frac{e^{i \alpha} \frac{z f^{\prime}(z)}{f(z)}+\lambda}{e^{i \alpha} \frac{z f^{\prime}(z)}{f(z)}+(2 \beta-\lambda)}\right| & =\left|\frac{\left(-p e^{i \alpha}+\lambda\right)+\sum_{n=1-p}^{\infty}\left(n e^{i \alpha}+\lambda\right) a_{n} z^{n+p}}{\left(-p e^{i \alpha}+2 \beta-\lambda\right)+\sum_{n=1-p}^{\infty}\left(n e^{i \alpha}+2 \beta-\lambda\right) a_{n} z^{n+p}}\right| \\
& <\frac{\left|p e^{i \alpha}-\lambda\right|+\sum_{n=1-p}^{\infty}\left|n e^{i \alpha}+\lambda\right|\left|a_{n}\right|}{\left|p e^{i \alpha}-2 \beta+\lambda\right|-\sum_{n=1-p}^{\infty}\left|n e^{i \alpha}+2 \beta-\lambda\right|\left|a_{n}\right|} \\
& \leqq 1 .
\end{aligned}
$$

Therefore, if $f$ satisfies the coefficient estimate (2.34), then we know that $f$ satisfies the inequality (2.35). This completes the proof of Theorem 4.

Corollary 6 Iff $\in \mathcal{M C}_{p}(\alpha, \beta)$ satisfies the inequality

$$
\sum_{n=1-p}^{\infty}|n|\left(\left|n e^{i \alpha}+\lambda\right|+\left|n e^{i \alpha}+2 \beta-\lambda\right|\right)\left|a_{n}\right| \leqq p\left(\left|p e^{i \alpha}-2 \beta+\lambda\right|-\left|p e^{i \alpha}-\lambda\right|\right)
$$

for some real $\alpha, \beta$ and $\lambda(0 \leqq \lambda \leqq p \cos \alpha)$, then $f \in \mathcal{M C}_{p}(\alpha, \beta)$.

We need the following lemma to prove our next theorem.

Lemma 2 (See [11]) Let $\varphi$ be a nonconstant regular function in $\mathbb{U}$. If $|\varphi|$ attains its maximum value on the circle $|z|=r<1$ at $z_{0}$, then

$$
z_{0} \varphi^{\prime}\left(z_{0}\right)=k \varphi\left(z_{0}\right)
$$

where $k \geqq 1$ is a real number.

Theorem 5 Iff $\in \mathcal{M S}_{p}(0, \beta)$ satisfies

$$
\left|1+\frac{z f^{\prime \prime}(z)}{f^{\prime}(z)}-\frac{z f^{\prime}(z)}{f(z)}\right|<\frac{\beta-p}{2 \beta} \quad(z \in \mathbb{U})
$$

for some real $\beta>p$, then $f \in \mathcal{M S}_{p}(0, \beta)$.

Proof Let us define the function $\phi$ by

$$
\phi(z):=\frac{\frac{z f^{\prime}(z)}{f(z)}+p}{\frac{z f^{\prime}(z)}{f(z)}+2 \beta-p} \quad(z \in \mathbb{U}),
$$

then we see that $\phi$ is analytic in $\mathbb{U}$ and $\phi(0)=0$. It follows from (2.39) that

$$
\frac{z f^{\prime}(z)}{f(z)}=\frac{-p+(2 \beta-p) \phi(z)}{1-\phi(z)}
$$

Differentiating both sides of (2.40) logarithmically, we obtain

$$
1+\frac{z f^{\prime \prime}(z)}{f^{\prime}(z)}-\frac{z f^{\prime}(z)}{f(z)}=\frac{(2 \beta-p) z \phi^{\prime}(z)}{-p+(2 \beta-p) \phi(z)}+\frac{z \phi^{\prime}(z)}{1-\phi(z)} .
$$


By virtue of (2.38) and (2.41), we find that

$$
\left|1+\frac{z f^{\prime \prime}(z)}{f^{\prime}(z)}-\frac{z f^{\prime}(z)}{f(z)}\right|=\left|\frac{2(\beta-p) z \phi^{\prime}(z)}{[-p+(2 \beta-p) \phi(z)][1-\phi(z)]}\right|<\frac{\beta-p}{2 \beta} .
$$

Suppose that there exists a point $z_{0} \in \mathbb{U}$ such that

$$
\max _{|z| \leqq\left|z_{0}\right|}|\phi(z)|=\left|\phi\left(z_{0}\right)\right|=1
$$

Then, Lemma 2 gives us that $\phi\left(z_{0}\right)=e^{i \theta}$ and $z_{0} \phi^{\prime}\left(z_{0}\right)=k e^{i \theta}(k \geqq 1)$. For such a point $z_{0}$, we have that

$$
\begin{aligned}
\left|1+\frac{z_{0} f^{\prime \prime}\left(z_{0}\right)}{f^{\prime}\left(z_{0}\right)}-\frac{z_{0} f^{\prime}\left(z_{0}\right)}{f\left(z_{0}\right)}\right| & =\left|\frac{2(\beta-p) k e^{i \theta}}{\left[-p+(2 \beta-p) e^{i \theta}\right]\left[1-e^{i \theta}\right]}\right| \\
& =\frac{2(\beta-p) k}{\sqrt{p^{2}+(2 \beta-p)^{2}-2 p(2 \beta-p) \cos \theta} \sqrt{2-2 \cos \theta}} \\
& \geqq \frac{\beta-p}{2 \beta} .
\end{aligned}
$$

This contradicts our condition (2.38). Therefore, there is no $z_{0} \in \mathbb{U}$ such that $\left|\phi\left(z_{0}\right)\right|=1$. This implies that $|\phi(z)|<1\left(z \in \mathbb{U}^{*}\right)$, that is,

$$
\left|\frac{\frac{z f^{\prime}(z)}{f(z)}+p}{\frac{z f^{\prime}(z)}{f(z)}+(2 \beta-p)}\right|<1 \quad(z \in \mathbb{U}) .
$$

Thus, we conclude that $f \in \mathcal{M S}_{p}(0, \beta)$.

Theorem 6 If $\in \mathcal{M S}_{p}(0, \beta)$ for some real $p<\beta \leqq p+\frac{1}{2}$, then

$$
\Re\left(\frac{1}{z^{p} f(z)}\right)>\frac{1}{1-2 \beta+2 p} \quad(z \in \mathbb{U})
$$

Proof Consider the function $\eta$ such that

$$
\frac{1}{z^{p} f(z)}=\frac{1+(1-2 \gamma) \eta(z)}{1-\eta(z)}
$$

for $\gamma=\frac{1}{1-2 \beta+2 p}$ and $f(z) \in \mathcal{M S}_{p}(0, \beta)$. Then we know that

$$
\Re\left(-\frac{z f^{\prime}(z)}{f(z)}\right)=\Re\left(p+\frac{(1-2 \gamma) z \eta^{\prime}(z)}{1+(1-2 \gamma) \eta(z)}+\frac{z \eta^{\prime}(z)}{1-\eta(z)}\right)<\beta .
$$

Since $\eta(z)$ is analytic in $\mathbb{U}$ and $\eta(0)=0$, we suppose that there exists a point $z_{0} \in \mathbb{U}$ such that

$$
\max _{|z| \leqq\left|z_{0}\right|}|\eta(z)|=\left|\eta\left(z_{0}\right)\right|=1 .
$$


Then, applying Lemma 2, we can write that $\eta\left(z_{0}\right)=e^{i \theta}$ and $z_{0} \eta^{\prime}\left(z_{0}\right)=k e^{i \theta}(k \geqq 1)$. This gives us that

$$
\begin{aligned}
\Re\left(-\frac{z_{0} f^{\prime}\left(z_{0}\right)}{f\left(z_{0}\right)}\right) & =\Re\left(p+\frac{(1-2 \gamma) k e^{i \theta}}{1+(1-2 \gamma) e^{i \theta}}+\frac{k e^{i \theta}}{1-e^{i \theta}}\right) \\
& \geqq p-\frac{(1-2 \gamma) k}{2 \gamma}-\frac{k}{2} \\
& \geqq p+\frac{\gamma-1}{2 \gamma}=\beta,
\end{aligned}
$$

which contradicts the inequality (2.46). Therefore, there is no $z_{0} \in \mathbb{U}$ such that $\left|\eta\left(z_{0}\right)\right|=1$. This means that $|\eta(z)|<1$, and that

$$
\Re\left(\frac{1}{z^{p} f(z)}\right)>\frac{1}{1-2 \beta+2 p} \quad(z \in \mathbb{U}) .
$$

The proof of Theorem 6 is thus completed.

In view of Theorem 6, we get the following result.

Corollary 7 Iff $\in \mathcal{M C}_{p}(0, \beta)$ for some real $p<\beta \leqq p+\frac{1}{2}$, then

$$
\Re\left(\frac{p}{z^{p+1} f^{\prime}(z)}\right)>\frac{1}{1-2 \beta+2 p} \quad(z \in \mathbb{U}) .
$$

\section{Competing interests}

The authors declare that they have no competing interests.

\section{Authors' contributions}

The authors jointly worked on deriving the results and approved the final manuscript.

\section{Author details}

${ }^{1}$ School of Mathematics and Statistics, Anyang Normal University, Anyang, Henan 455002, People's Republic of China. ${ }^{2}$ School of Railway Tracks and Transportation, East China Jiaotong University, Nanchang, Jiangxi 330013, People's Republic of China.

\section{Acknowledgements}

The present investigation was supported by the National Natural Science Foundation under Grant 11226088 and the Key Project of Natural Science Foundation of Educational Committee of Henan Province under Grant 12A110002 of the People's Republic of China. The authors are grateful to the referees for their valuable comments and suggestions which essentially improved the quality of the paper.

Received: 20 November 2012 Accepted: 9 July 2013 Published: 23 July 2013

\section{References}

1. Wang, Z-G, Sun, Y, Zhang, Z-H: Certain classes of meromorphic multivalent functions. Comput. Math. Appl. 58, 1408-1417 (2009)

2. Uyanik, N, Shiraishi, H, Owa, S, Polatoglu, Y: Reciprocal classes of $p$-valently spirallike and $p$-valently Robertson functions. J. Inequal. Appl. 2011, Article ID 61 (2011)

3. Ali, RM, Ravichandran, V: Classes of meromorphic $\alpha$-convex functions. Taiwan. J. Math. 14, 1479-1490 (2010)

4. Aouf, MK: Certain subclasses of meromorphically multivalent functions associated with generalized hypergeometric function. Comput. Math. Appl. 55, 494-509 (2008)

5. Aouf, MK: Certain subclasses of meromorphically $p$-valent functions with positive or negative coefficients. Math. Comput. Model. 47, 997-1008 (2008)

6. Silverman, H, Suchithra, K, Stephen, BA, Gangadharan, A: Coefficient bounds for certain classes of meromorphic functions. J. Inequal. Appl. 2008, Article ID 931981 (2008)

7. Srivastava, HM, Owa, S: Current Topics in Analytic Function Theory. World Scientific, Singapore (1992) 
8. Srivastava, HM, Patel, J: Some subclasses of meromorphically multivalent functions associated with a linear operator. Appl. Math. Comput. 195, 11-23 (2008)

9. Wang, Z-G, Jiang, Y-P, Srivastava, HM: Some subclasses of meromorphically multivalent functions associated with the generalized hypergeometric function. Comput. Math. Appl. 57, 571-586 (2009)

10. Wang, Z-G, Liu, Z-H, Xiang, R-G: Some criteria for meromorphic multivalent starlike functions. Appl. Math. Comput. 218, 1107-1111 (2011)

11. Jack, IS: Functions starlike and convex of order $\alpha$. J. Lond. Math. Soc. 3, 469-474 (1971)

doi:10.1186/1029-242X-2013-336

Cite this article as: Shi et al.: Some subclasses of multivalent spirallike meromorphic functions. Journal of Inequalities and Applications 2013 2013:336.

Submit your manuscript to a SpringerOpen ${ }^{\circ}$ journal and benefit from:

- Convenient online submission

- Rigorous peer review

- Immediate publication on acceptance

- Open access: articles freely available online

- High visibility within the field

- Retaining the copyright to your article

Submit your next manuscript at $>$ springeropen.com 